\title{
Microwave assisted extraction of flavonoids from pomegranate peel and its antioxidant activity
}

\author{
Ju Huang ${ }^{\text {a }}$, Weiping $\mathrm{He}^{2}$, Chen Yan ${ }^{1}$, Xihua $\mathrm{Du}^{1}$ and Xiaoqin Shi ${ }^{1}$ \\ ${ }^{1}$ School of Chemistry \& Chemical Engineering, Xuzhou Institute of Technology, Xuzhou 221111, Jiangsu, China \\ ${ }^{2}$ School of Chemical Engineering, Xuzhou College of Industrial Technology, Xuzhou 221140, Jiangsu, China
}

\begin{abstract}
Pomegranate peel, a waste generated from fruit processing industry, which contains maximum amounts of bioactive compounds than the juice, is a potential source of active ingredient such as flavonoids that are known for their antioxidant properties. Microwaveassisted extraction (MAE) of flavonoids from pomegranate peel and its antioxidant activity were investigated. Three independent and main variables, including ethanol concentration(\%), solvent/solid ratio(mL/g) and MAE time(min), which were of significance for the yields of flavonoids were studied. The optimal conditions were determined by the orthogonal design. The maximum extraction yield of $4.260 \%$ was achieved at ethanol concentration of $60 \%$, ratio of solvent to material of 40:1 and MAE time of $3 \mathrm{~min}$. In vitro antioxidant assays showed that the extract exhibited a strong DPPH radical-scavenging ability with $\mathrm{IC}_{50}$ value of $0.187 \mathrm{mg} / \mathrm{mL}$. Results indicated that MAE was a suitable approach for the extraction of flavonoids from pomegranate peel.
\end{abstract}

\section{Introduction}

Pomegranate (Punica granatum L.) is a shrub or a small tree widely distributed in both tropical and subtropical areas. It is an important source of bioactive compounds and has been used for folk medicine for many centuries [1]. It has nutritional and medical benefits such as antioxidantive, anticancer, and antimutagenic efficacy. Many researches indicate that the Pomegranate peels contain much more bioactive compounds than the juice, which are one of the most valuable by-products of the food industry [2-4].

Compared with the traditional methods, MAE has many advantages, such as higher extraction rate, shorter extraction time, use of less solvent, better productivity and higher quality products. It is a widely used technique for the extraction of valuable bioactive components from plant materials. In microwave assisted solid-liquid extraction, the extraction is accelerated due to the changes in cell anatomy caused by the impact of microwave radiation. The accelerated extraction may be attributed to synergistic action of temperature and concentration gradients [5-7].

The objective of this study was to optimise the MAE conditions for the extraction of flavonoids from Pomegranate peel. The orthogonal design was employed to study the optimal ethanol

\footnotetext{
${ }^{a}$ Corresponding author: 281770895@qq.com.

This work was supported by the research projects of college(No. XKY2013317), Xuzhou institute of technology, China.
} 
concentration (\%), solvent/solid ratio $(\mathrm{mL} / \mathrm{g})$ and MAE time $(\mathrm{min})$ which could maximise the yield of flavonoids from Pomegranate peel. Then, the colorimetric method with $\mathrm{AlCl}_{3} /$ ethanol system was used to quantify total flavonoids. In addition, the antioxidant activity of flavonoids from Pomegranate peel, obtained under optimised MAE extraction conditions was determined by 2,2-diphenyl-1picrylhydrazyl (DPPH) radical-scavenging assay.

\section{Materials and methods}

\subsection{Raw materials and reagents}

Pomegranate peels were obtained from the fresh fruit purchased from the local market in Xuzhou City, Jiangsu Province,China. The peels were dried in an oven with air circulation at $60^{\circ} \mathrm{C}$ and then ground into a fine powder(40-60 mesh).

Rutin was purchased from Aladdin reagents, 2,2-diphenyl-1-picrylhydrazyl (DPPH) and ascorbid $\operatorname{acid}(\mathrm{Vc})$ were purchased from sigma. All the other reagents were of analytical grade.

\subsection{Instruments}

UV-VIS spectrophotometer T6, Beijing PERSEE General Instrument Co., Ltd. A microwave oven P70F20L-DG(S0), Galanz microwave life Electric Appliance Co., Ltd. Electronic balance JA2003, Shanghai Precision Scientific Instrument Co., Ltd. Blower drying box DHG-9070A, Suzhou Jiangdong Precision Instrument Co. Ltd.

\subsection{Microwave assisted extraction and experimental design}

A modified microwave oven (P70F20L-DG(S0), Galanz, China) was used for microwave assisted extraction. The system was equipped with a temperature sensor for monitoring and regulating the internal temperature of the extraction vessels. Dry samples $(1 \mathrm{~g})$ were placed into $50 \mathrm{~mL}$ beaker extraction vessels and the solvent added. The container was capped, and the system was started-up. The exact temperature was detected by a sensor. When the desired temperature was reached, the heating device would automatically shut down for a while until the temperature dropped. The extraction temperature and time were set at different degrees according to different conditions. After extraction, the vessels were left for several minutes to cool down to room temperature.

In order to optimize microwave assisted extraction (MAE) of antioxidant compounds from Pomegranate peels, especially flavonoids, different ethanol concentration (40-80\%), solvent/solid ratio(10-50mL/g) and MAE time(1-5min) were evaluated individually. The results of the single factor analysis were combined together in order to maximize the extraction in terms of flavonoids yield and antioxidant activity with orthogonal experiments.

\subsection{Determination of flavonoids content and yield of flavonoids}

The content of flavonoids was determined by spectrophotometry using the aluminium chloride colorimetric method with some modifications [8]. Briefly, $2 \mathrm{~mL}$ diluted solution containing flavonoids was added to a $25 \mathrm{~mL}$ volumetric flask, and $1 \mathrm{~mL}$ of $5 \%(\mathrm{w} / \mathrm{w}) \mathrm{NaNO}_{2}$ was mixed for 6 min, and then $1 \mathrm{~mL}$ of $10 \% \mathrm{AlCl}_{3}(\mathrm{w} / \mathrm{w})$ was added and mixed, 6 min later, $10 \mathrm{~mL}$ of $1 \mathrm{~mol} / \mathrm{L} \mathrm{NaOH}$ was added. After $15 \mathrm{~min}$, the absorbance of the solution at $400 \mathrm{~nm}$ was measured with uv/vis spectrophotometer (T6, PGENENAL, Beijing, China) against the same mixture, without the sample as a blank. The calibration curve was prepared by preparing Rutin solutions at concentrations from 0 to $0.1 \mathrm{mg} / \mathrm{mL}$ in ethanol. It was determined to be as the following: $A=7.7750 c+0.0176$, where $A$ is absorbance value of sample, $c$ is sample concentration $\left(\mathrm{R}^{2}=0.9945\right)$.

The extraction yield of flavonoids was expressed according to Eq. (1): 
Yield of flavonoids

$$
Y=\frac{C \times N \times V}{m} \times 100 \%
$$

Where $C$ is mass concentration of flavonoids, $N$ is dilute times, $V$ is the volume of the extraction liquid, $m$ is sample mass(Pomegranate peels).

\subsection{Determination of antioxidant activity}

The scavenging activity of flavonoid from Pomegranate peels towards DPPH-radical was measured according to the method by Xie[7], with some modifications. Briefly, $0.2 \mathrm{mmol} / \mathrm{L}$ solution of DPPH in ethanol was prepared daily before measurements, $2 \mathrm{~mL}$ of various concentrations $(0.1,0.2,0.3,0.4,0.5$, $0.6,0.7,0.8,0.9$ and $1.0 \mathrm{mg} / \mathrm{g}$ ) of the sample solutions were thoroughly mixed with $2 \mathrm{~mL}$ of freshly prepared DPPH and $1 \mathrm{~mL}$ ethanol. The mixture was shaken vigorously and allowed to stand for $30 \mathrm{~min}$ in the dark, and the absorbance at $518 \mathrm{~nm}$ was then measured against a blank with uv/vis spectrophotometer (T6, PGENENAL, Beijing, China). A lower absorbance value of the reaction mixture indicates a higher free radical scavenging activity. Ethanol was used as the blank control, ascorbic acid was used as positive controls. All tests were carried out in triplicate. The capability to scavenge the free radical DPPH in percentage of sample (I\%) was calculated according to the following equation.

$$
I \%=\left(1-\frac{A_{i}-A_{j}}{A_{o}}\right) \times 100 \%
$$

Where $A_{0}$ is the absorbance of the incubation DPPH solution without addition of the sample or positive controls, $A_{i}$ is the absorbance of the incubation mixture containing both the test sample and DPPH solution and $A_{j}$ is the absorbance of the sample without DPPH solution. The concentration of sample producing a $50 \%$ reduction of the radical absorbance $\left(I C_{50}\right)$ was used as an index to compare the antioxidant activity.

\section{Result and discussion}

\subsection{Effect of ethanol concentration, solvent/solid ratio and MAE time}

Experiments were conducted to study the effect of ethanol concentration on the yield of flavonoids from Pomegranate peels. The extraction was performed at 40, 50,60, 70and 80\% respectively, the extraction time was fixed to $3 \mathrm{~min}$, the ratio of solvent to material was fixed to $20: 1$, and the other conditions were kept the same. Figure 1(a) shows the effect of different ethanol concentration on extraction of total flavonoids. The results showed that the yield increased rapidly at first, then decreased when ethanol concentration exceeds $60 \%$. So the optimized ethanol concentration is $60 \%$ for further experiments.

The effect of the ratio of solvent to material on the MAE process was carried out at 10:1, 20:1, 30:1, 40:1 and 50:1,respectively, while the other experimental conditions were as follows: extraction time of $3 \mathrm{~min}$, and solvent of $60 \%(\mathrm{v} / \mathrm{v})$ ethanol. As shown in Figure 1(b) the yield of flavonoids first increased then decreased with increasing the ratio of solvent to raw material, where an optimized ratio is $30: 1 \sim 40: 1 \mathrm{~mL} / \mathrm{g}$. In general, a higher ratio of solvent to raw material could result in larger concentration gradient during the diffusion from solid into the solution, and a igher extraction yield. On the other hand, too much solvent would decrease the microwave adsorption of material, since more energy was needed to be absorbed by the solvent. Therefore, the breakage of material cell wall and mass transmission might be negatively influenced.Thus, the ratio of solvent to raw material of 30:1 40:1 $\mathrm{mL} / \mathrm{g}$ was adopted in the present work. 
The extraction yield of flavonoids affected by different extraction time is shown in Figure 1(c) when other extraction conditions were as follows: ethanol concentration of $60 \%$ and ratio of solvent to material weight of $30: 1 \mathrm{~mL} / \mathrm{g}$, respectively. The extraction yield of flavonoids shows the trend of first increase then decrease with increasing the extraction time, where a maximum yield $(4.1 \%)$ was obtained at the extraction time of $3 \mathrm{~min}$. MAE is a fast extraction process where microwave energy is delivered efficiently to materials through molecular interaction under the electromagnetic field, offering a rapid transfer of energy to the extraction solvent and plant materials. However, long extraction time induced the degradation of flavonoids, and the yield of extraction decreased. Therefore, 3 min was selected as the center point for further experiments.
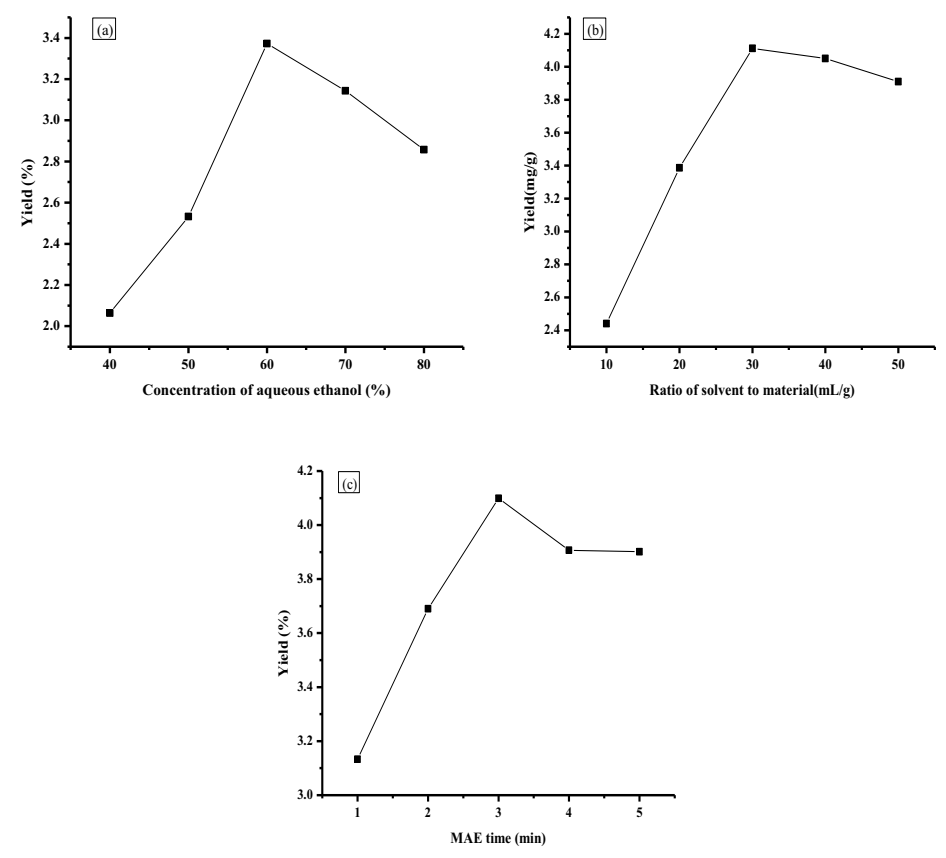

Figure 1. The effects of (a) concentration of aqueous ethanol, (b) Ratio of solvent to material and (c) MAE time on the extraction yields.

According to the single-factor experiments, we adopted ethanol concentration $50-60-70 \%$, the ratio of solvent to material 20:1-30:1-40:1 $\mathrm{mL} / \mathrm{g}$ and extract time 2-3-4 $\mathrm{min}$ for orthogonal experiments.

\subsection{Optimization of MAE operating parameters by orthogonal experiment}

The yield of flavonoids at different expermental combinations were given in Table 1. The results showed that the effect on the yield of total flavonoids was decreased in the following order when MAE was used to extract total flavonoids from Pomegranate peels: ethanol concentration, the ratio of solvent to material and MAE time, that is $\mathrm{A}>\mathrm{B}>\mathrm{C}$. The optimal conditions for flavonoids extract from Pomegranate peels are ethanol concentration of $60 \%$, ratio of solvent to material of 40:1 and MAE time of $3 \mathrm{~min}$. 
Table 1. Experimental design runs in orthogonal method $\mathrm{L}_{9}\left(3^{4}\right)$ and the observed value of extraction yield.

\begin{tabular}{|c|c|c|c|c|c|}
\hline \multirow{2}{*}{$\begin{array}{c}\text { Experiment } \\
\text { number }\end{array}$} & $\mathbf{A}$ & B & C & D & \multirow[b]{2}{*}{ yield $(\%)$} \\
\hline & $\begin{array}{c}\text { ethanol } \\
\text { concentration }(\%)\end{array}$ & $\begin{array}{l}\text { solvent/solid } \\
\text { ratio }(\mathrm{mL} / \mathrm{g})\end{array}$ & $\begin{array}{c}\text { MAE } \\
\text { time(min) }\end{array}$ & $\begin{array}{l}\text { blank } \\
\text { column }\end{array}$ & \\
\hline 1 & 50 & 20 & 2 & 1 & 2.946 \\
\hline 2 & 50 & 30 & 3 & 2 & 3.256 \\
\hline 3 & 50 & 40 & 4 & 3 & 3.171 \\
\hline 4 & 60 & 20 & 3 & 3 & 3.586 \\
\hline 5 & 60 & 30 & 4 & 1 & 3.978 \\
\hline 6 & 60 & 40 & 2 & 2 & 4.186 \\
\hline 7 & 70 & 20 & 4 & 2 & 3.590 \\
\hline 8 & 70 & 30 & 2 & 3 & 3.684 \\
\hline 9 & 70 & 40 & 3 & 1 & 4.126 \\
\hline $\mathrm{k}_{1}$ & 1.039 & 1.125 & 1.202 & 1.228 & \\
\hline $\mathrm{k}_{2}$ & 1.306 & 1.211 & 1.216 & 1.224 & \\
\hline $\mathrm{k}_{3}$ & 1.267 & 1.276 & 1.193 & 1.160 & \\
\hline $\mathrm{R}$ & 0.799 & 0.476 & 0.069 & 0.203 & \\
\hline
\end{tabular}

\subsection{Analysis of variance and verification}

The interactions and coefficients on the orthogonal experiments were obtained by analysis of variance (ANOVA) (Table 2). The results show that factor A is very significant, while B and C are not.

Table 2. ANOVA for the MAE extraction conditions.

\begin{tabular}{|c|c|c|c|c|c|}
\hline factor & $\begin{array}{c}\text { the sum of } \\
\text { variances }\end{array}$ & $\begin{array}{c}\text { degrees of } \\
\text { freedom }\end{array}$ & variance & F-value & significant \\
\hline A & 1.118 & 2 & 0.559 & 14.333 & $*$ \\
\hline B & 0.311 & 2 & 0.1555 & 3.987 & \\
\hline C & 0.007 & 2 & 0.0035 & 0.09 & \\
\hline D & 0.078 & 2 & 0.039 & 1 & \\
\hline error & 0.08 & 8 & & & \\
\hline
\end{tabular}

In order to validate the reliability of the optimum MAE conditions, five experiments were executed at the optimal conditions. The experimental yield of flavonoids from Pomegranate peels was $4.260 \%(\mathrm{n}=5, \mathrm{RSD}<0.17)$. 


\subsection{Antioxidant activity}

The antioxidant activity of flavonoids from Pomegranate peels was assessed with DPPH-scavenging assay, in comparison with known antioxidants ascorbic acid (Figure 2). It was observed that the flavonoids from Pomegranate peels exhibited notable DPPH radical-scavenging activity, and the DPPH radical scavenging effects were increased with increasing flavonoids concentrations. It increased from $31.75 \%$ to $92.85 \%$ when the concentrations of the flavonoids increased from 0.109 $\mathrm{mg} / \mathrm{mL}$ to $0.886 \mathrm{mg} / \mathrm{mL}$. However, DPPH free radical-scavenging of flavonoids from Pomegranate peels was less than that of $\mathrm{VC}$, but the results indicated that flavonoids extracted by MAE had a strong DPPH radical-scavenging activity, with an $\mathrm{IC}_{50}$ value of $0.187 \mathrm{mg} / \mathrm{mL}$.

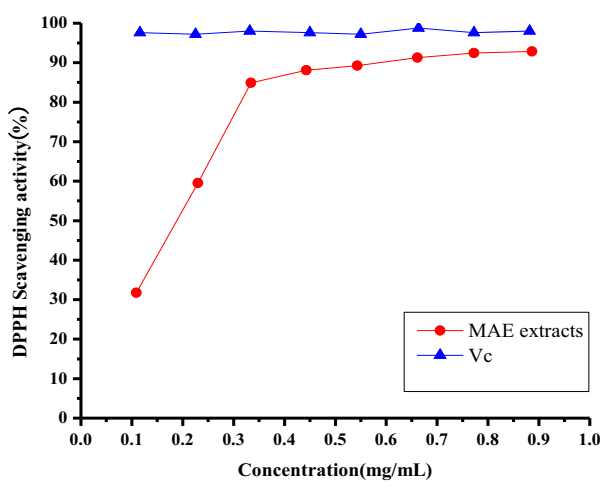

Figure 2. Antioxidant activity of flavonoids from pomegranate peel assessed by DPPH radical scavenging assay.

\section{Conclusion}

Microwave irradiation, which has proved to be a clean, efficient and convenient energy source, has been utilised in the extraction of flavonoids from Pomegranate peels. Orthogonal experiment was successfully applied for optimisation of MAE of flavonoids from Pomegranate peels. The optimal flavonoids yield of $4.26 \%$ was obtained when the optimum conditions (ethanol concentration of $60 \%$, ratio of solvent to material of 40:1 and MAE time of 3min.) were used. Application of MAE in the extraction of flavonoids from Pomegranate peels dramatically reduced extraction time. The flavonoids obtained by MAE exhibited good antioxidant activity with an $\mathrm{IC}_{50}$ value of $0.187 \mathrm{mg} / \mathrm{mL}$. Thus, MAE was proposed as an alternative for the extraction of flavonoids from Pomegranate peels, and orthogonal design was an useful tool for the optimisation of MAE process of flavonoids from Pomegranate peels.

\section{References}

1. Wang C, Shi L, Fan L, Ding Y, Zhao S, Liu Y, et al. Ind Crops Prod.42,587(2013).

2. Sood A, Gupta M. Food Bios. 12,100(2015).

3. Zhu C, Liu X. Carbohydr Polym. 92(2),1197(2013).

4. Amyrgialaki E, Makris DP, Mauromoustakos A, Kefalas P. Ind Crops Prod.59,216 (2014).

5. Thirugnanasambandham K, Sivakumar V, Maran JP. Int J Biol Macromol.72,1( 2015).

6. Bouras M, Chadni M, Barba FJ, et al. Ind Crops Prod. 77,590 (2015)

7. Xie JH, Dong CJ, Nie SP, Li F, Wang ZJ, Shen MY, et al. Food Chem. 186,97-105(2015).

8. Amir, M., Mujeeb, M., Khan, A., Ashraf, K., Sharma, D., \& Aqil, M. International Journal of Green Pharmacy, 6, 67-72 (2012). 